\title{
A high energy nanosecond cryogenic cooled Yb:YAG active-mirror amplifier system
}

\author{
Xiaojin Cheng ${ }^{1}$, Jianlei Wang ${ }^{1}$, Zhongguo Yang ${ }^{1}$, Jin Liu ${ }^{1}$, Lei $\mathrm{Li}^{1}$, Xiangchun $\mathrm{Shi}^{1}$, Wenfa Huang ${ }^{2}$, \\ Jiangfeng Wang ${ }^{2}$, and Weibiao Chen ${ }^{1}$ \\ ${ }^{1}$ Shanghai Key Laboratory of All Solid-state Laser and Applied Techniques, Shanghai Institute of Optics and Fine Mechanics, \\ Chinese Academy of Sciences, Shanghai 201800, China \\ ${ }^{2}$ The Joint Laboratory for High Power Laser Physics, Shanghai Institute of Optics and Fine Mechanics, Chinese Academy of Sciences, \\ Shanghai 201800, China \\ (Received 3 March 2014; revised 9 May 2014; accepted 22 May 2014)
}

\begin{abstract}
A diode-pumped master oscillator power amplifier system based on a cryogenic Yb:YAG active-mirror laser has been developed. The performances of the laser amplifier at low temperature and room temperature have been investigated theoretically and experimentally. A maximum output energy of $3.05 \mathrm{~J}$ with an optical-to-optical efficiency of $14.7 \%$ has been achieved by using the master amplifier system.
\end{abstract}

Keywords: diode-pumped; solid-state laser; Yb:YAG

\section{Introduction}

Solid-state lasers with high energy have been widely reported in many application fields, ranging from materials processing to remote sensing to laser-driven inertial fusion $^{[1]}$. For high energy laser oscillator and amplifier design, the amplified spontaneous emission (ASE), the thermal effects of the laser material, and the laser-induced damage threshold of the optics are the most important considerations. In addition to relations to the physical character, such as the quantum defect, concentration quenching, up-conversion, etc., the thermal effects of laser materials have great relations to the pump and cooling structure ${ }^{[2,3]}$. Prevention of damage to the optics can be achieved by scaling the laser gain medium and laser spot size. In order to suppress the ASE, reasonable design of the amplifier gain, the size and the shape of the gain medium is necessary.

The development of laser diodes has promoted the interest in $\mathrm{Yb}^{3+}$ doped laser gain media, such as $\mathrm{YAG}, \mathrm{CaF}_{2}$, $\mathrm{Y}_{2} \mathrm{O}_{3}$, and S-FAP ${ }^{[4-7]}$. In particular, $\mathrm{Yb}: \mathrm{YAG}$, with a long fluorescence lifetime, broad emission band, low quantum defect, and excellent thermo-mechanical properties, has been considered to have great potential as a material to obtain high energy, high efficiency, and short duration pulse laser output using diode-pumped solid-state laser (DPSSL) systems ${ }^{[8,9]}$.

Correspondence to: Xiao-jin Cheng, Shanghai Key Laboratory of All Solid-state Laser and Applied Techniques, Shanghai Institute of Optics and Fine Mechanics, Chinese Academy of Sciences, 201800, No. 390, Qinghe Road, Jiading District, Shanghai, China. Email: xjcheng@ siom.ac.cn
Moreover, to obtain a much larger laser gain medium, Yb:YAG ceramic has already attracted attention in the field of high power lasers. However, the performance of an Yb:YAG laser strongly depends on the temperature because of its quasi-three-level nature. At room temperature, the laser operation threshold of Yb:YAG crystal is high for the reabsorption of a lower energy level. Imposition of a high intensity pump or decrease of the laser material temperature will allow this problem to be overcome ${ }^{[10-12]}$.

Researchers have studied high energy DPSSL systems at several hertz repetition rates during the last decade, for example Mercury, Lucia, Halna, and Polaris. Mercury laser systems delivered $61 \mathrm{~J}(10 \mathrm{~Hz})$ at the Lawrence Livermore National Laboratory (LLNL) with an Yb:S-FAP crystal and cooled by high pressure helium flow ${ }^{[13]}$. With Yb:YAG crystal, the Lucia laser system delivered $14 \mathrm{~J}(2 \mathrm{~Hz})$ at the LULI laboratory ${ }^{[14,15]}$. The Halna laser system at the Institute for Laser Engineering (ILE) delivered $20 \mathrm{~J}(10 \mathrm{~Hz})$ with a zigzag Nd:glass slab ${ }^{[16]}$. For the Polaris laser system, $12 \mathrm{~J}(0.05 \mathrm{~Hz})$ was obtained from Yb:glass at the Institute of Optics and Quantum Electronics of the Friedrich-Schiller University (IOQ, Jena, Germany) ${ }^{[17-20]}$.

In this paper, in order to apply a high energy pump source with a Ti:sapphire laser to obtain ultrashort pulses, a diodepumped master oscillator power amplifier system based on a cryogenic Yb:YAG/YAG active-mirror laser has been set up for the first step. With a doping concentration of 4 at.\% for the Yb:YAG/YAG crystal and cooled by liquid nitrogen, 

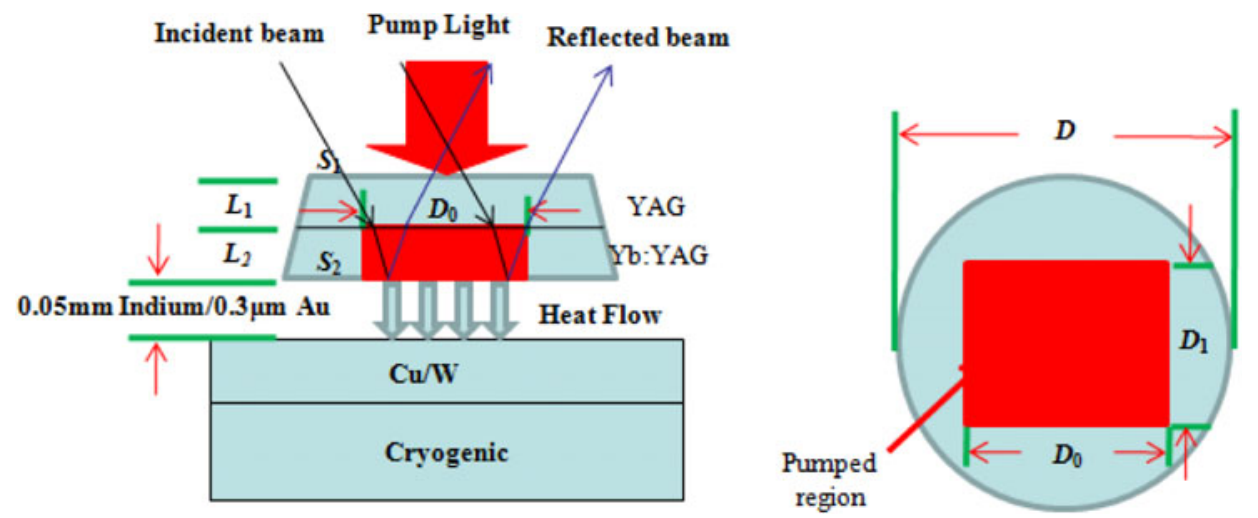

Figure 1. Illustration of the pump and cooling structure of the active-mirror amplifier with a cryogenic cooled composite Yb:YAG/YAG crystal.

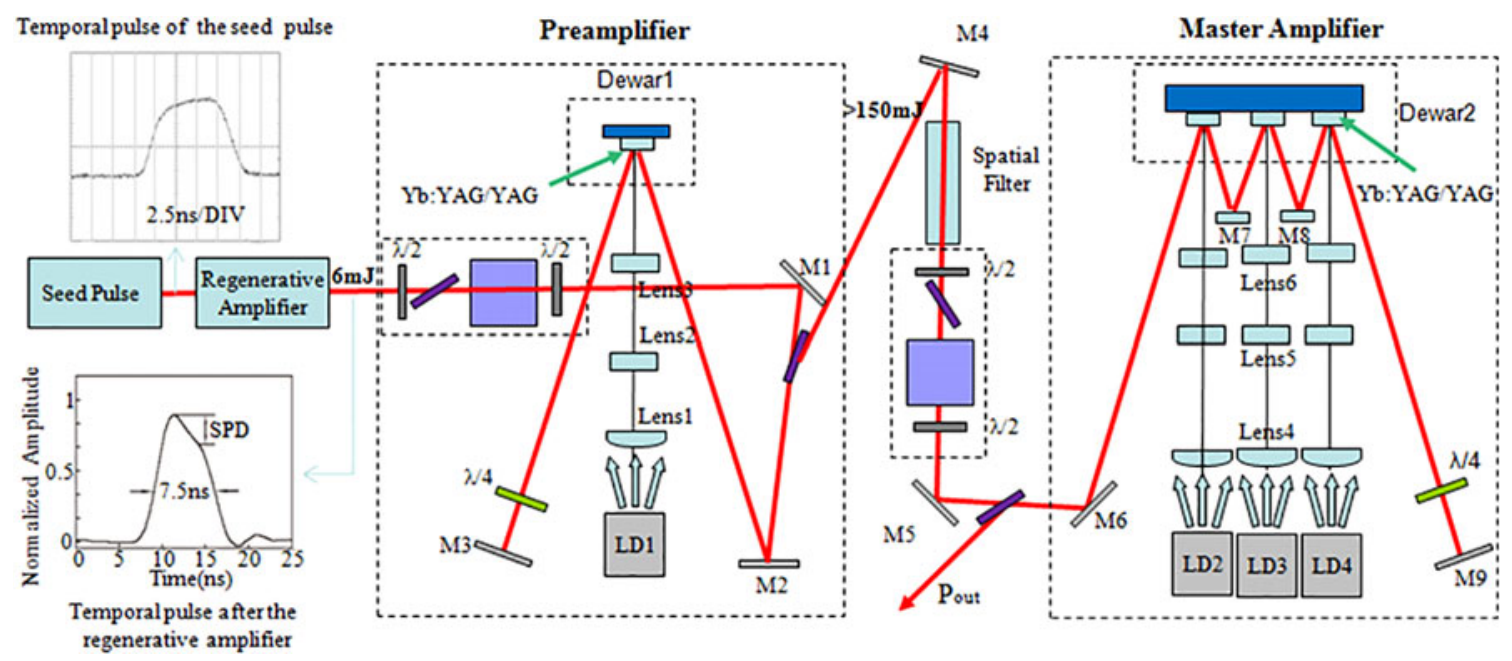

Figure 2. Scheme of the active-mirror amplifier setup.

$3.05 \mathrm{~J}$ at an optical-to-optical efficiency of $14.7 \%$ has been achieved at a cooling temperature of $155 \mathrm{~K}$.

\section{Experimental setup}

Figure 1 shows the pump and cooling structure of the active-mirror amplifier with cryogenic cooled composite Yb:YAG/YAG crystal. Facet $S_{1}$ is AR-coated at both $940 \pm$ $15 \mathrm{~nm}\left(0^{\circ}\right)$ and $1030 \pm 15 \mathrm{~nm}\left(15^{\circ}\right)$. The other end facet, $S_{2}$, is HR-coated at both $940 \pm 15 \mathrm{~nm}\left(0^{\circ}\right)$ and $1030 \pm 15 \mathrm{~nm}$ $\left(15^{\circ}\right)$ to reflect pump light and signal light. Meanwhile, facet $S_{2}$ is also used as the cooling surface and is wrapped in indium foil $(50 \mu \mathrm{m})$ and thin gold foil $(0.3 \mu \mathrm{m})$. The crystal is fixed in a copper heat sink which is cooled by liquid nitrogen and sealed in a vacuum Dewar.

A diode-pumped master oscillator power amplifier system based on a cryogenic $\mathrm{Yb}$ :YAG/YAG active-mirror structure has also been constructed for further study of the laser performance of $\mathrm{Yb}$ :YAG at low temperature (Figure 2). The seed pulses with a pulse duration of $10 \mathrm{~ns}$ were generated in a CW distributed-feedback Yb fiber laser following an acoustic-optic chopper and optical fiber amplifiers which produced nanosecond pulses with a $10 \mathrm{~Hz}$ repetition rate and amplified up to $6 \mathrm{~mJ}$ in a regenerative amplifier ${ }^{[21]}$. To prevent unnecessary back reflection from the amplifier stage, the regenerative amplifier was followed by an optical isolator system which consisted of two Brewster polarizers, one $45^{\circ}$ rotator and two half-wave plates (HWPs). The size of the laser beam was shaped to $5.5 \times 5.5(\mathrm{~mm})$ in order to ensure the best matching with the pump light. A four-pass preamplifier was realized using reflection mirrors (M2, M3) and a quarter-wave plate (QWP). The size of the composite Yb:YAG/YAG was $\Phi 25 \times 6.5(\mathrm{~mm})$. In addition, the thickness of the doping part was $3.5 \mathrm{~mm}$ with a doping concentration of 4 at.\%. The laser diode stack (LD1) with a maximum output power of $2.95 \mathrm{~kW}$ at a center wavelength of $940 \mathrm{~nm}$ was available as the pump source. Lens 1, made up of one plano-convex lens with a curvature radius of $165 \mathrm{~mm}$ and thickness of $5 \mathrm{~mm}$, was designed to compress the fast axis beam. Meanwhile, a plano-convex group (lens 2 and lens 3), 
with a curvature radius of $70 \mathrm{~mm}$ and thickness of $15 \mathrm{~mm}$, was used to shape the slow axis beam. All of these three lenses were AR-coated at $940 \pm 15$ and $1030 \pm 15 \mathrm{~nm}$. After four-pass amplification, the laser beam was output from the polarizer and expander two times. At the same time, a spatial filter system was used to filter the higher order mode laser. The same isolator system was loaded between the preamplifier and the master amplifier.

For the master amplifier, three Yb:YAG/YAG modules were sealed in one Dewar with the same pump and cooling structure. The laser diode stacks (LD3, LD4, and LD5) could deliver $6.5 \mathrm{~kW}$ output power. Lens 4 , made up of one plano-convex lens with a curvature radius of $220 \mathrm{~mm}$ and thickness of $7 \mathrm{~mm}$, was designed to compress the fast axis beam. Meanwhile, a plano-convex group (lens 5 and lens 6 ), with a curvature radius of $82 \mathrm{~mm}$ and thickness of $12 \mathrm{~mm}$, was used to shape the slow axis beam. All of these lenses were AR-coated at $940 \pm 15$ and $1030 \pm 15 \mathrm{~nm}$.

\section{Laser experiments and results}

Figure 3 shows the output energy versus the pump energy at different cooling temperatures for an injection energy of $6 \mathrm{~mJ}$. The spot size of the injection signal light is $5.5 \times 5.5(\mathrm{~mm})$. As shown in Figure 3, the gain is not obvious because of the reabsorption at room temperature and will increase with decrease of the cooling temperature until $155 \mathrm{~K}$. Below $155 \mathrm{~K}$, the gain no longer increases because of the lateral ASE. At $155 \mathrm{~K}$, a maximum output energy of $196 \mathrm{~mJ}$ is obtained with a pump energy of $2.95 \mathrm{~J}$ at $10 \mathrm{~Hz}$. In order to evaluate the results of the experiment, a simulation of the preamplifier was made according to the laser rate equations. In order to reduce the complexity, the lateral ASE was not considered in our calculation. As shown in Figure 3, the simulation results match the experimental results excellently for temperatures of 200,250 , and $300 \mathrm{~K}$. However, at $155 \mathrm{~K}$, the experimental result deviates further from the simulation result with increase of the pump energy, which indicates that the lateral ASE is serious at $155 \mathrm{~K}$.

Figure 4 shows the output energy of the master amplifier after four-pass amplification. In order to reduce the influence of lateral ASE and parasitic oscillation, we roughened the side of the Yb:YAG crystal at the master amplifier. Before the signal pulse was injected into the master amplifier, the laser beam was expanded to $11 \times 11(\mathrm{~mm})$. At $155 \mathrm{~K}$, a maximum output energy of $3.05 \mathrm{~J}$ at an optical-to-optical efficiency of $14.7 \%$ was achieved for an injected pulse energy of $180 \mathrm{~mJ}$. To evaluate the scaling amplification of the active-mirror structure amplifier, a simulation was made for the master amplifier based on the laser rate equations. As described in Figure 4, for the same cooling temperature, the experimental result is slightly lower than the simulation result, the same growth can be obtained and the result shows that the output energy can increase linearly with a much more powerful pump.

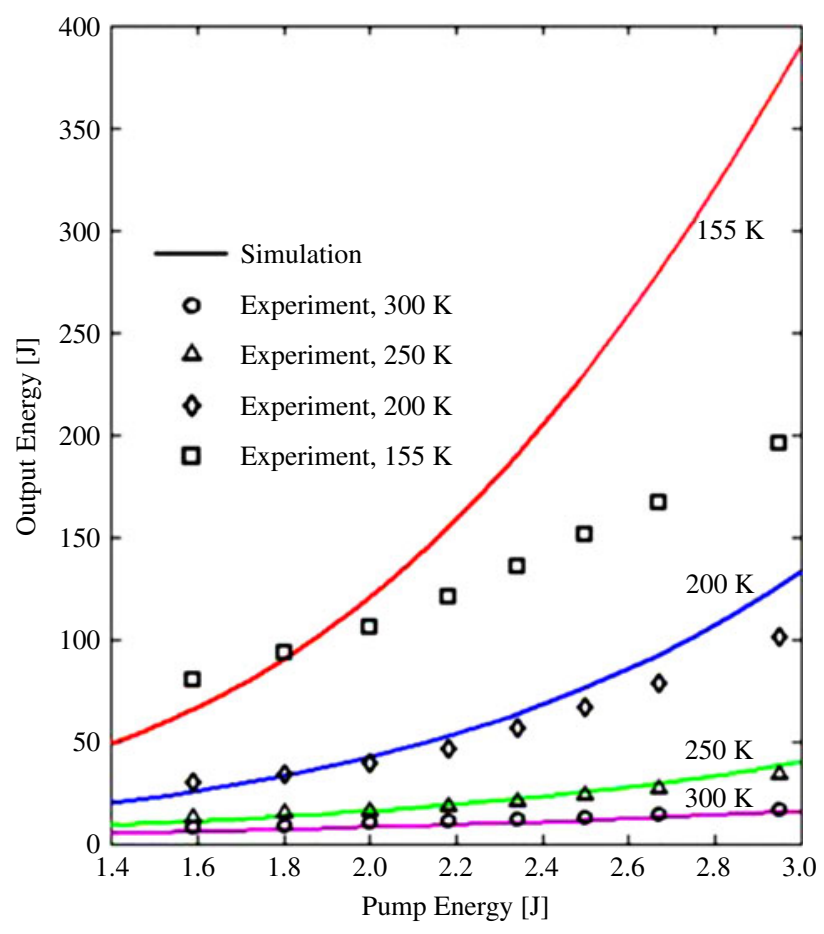

Figure 3. The output pulse energy of the preamplifier after four-pass amplification independent of the pump energy at a $10 \mathrm{~Hz}$ repetition rate at different cooling temperatures.

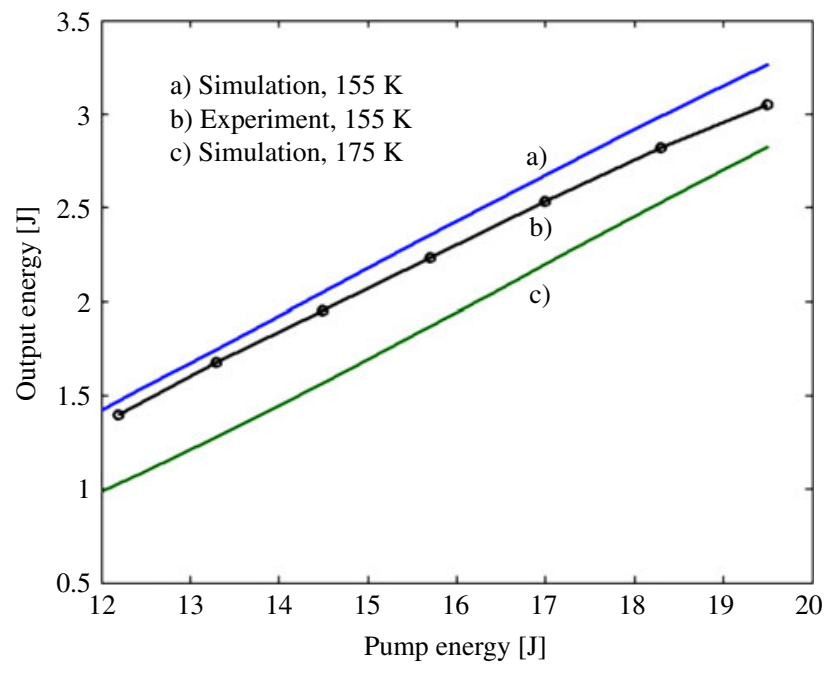

Figure 4. The measured and simulated pulse energy of the master amplifier after four-pass amplification independent of the pump energy at a $10 \mathrm{~Hz}$ repetition rate and injected pulse energy of $180 \mathrm{~mJ}$.

\section{Conclusion and outlook}

In conclusion, we have shown nanosecond pulse amplification to the $3.05 \mathrm{~J}$ level at a repetition rate of $10 \mathrm{~Hz}$. The seed pulses with a pulse duration of $10 \mathrm{~ns}$ were generated in an $\mathrm{Yb}$ fiber laser and amplified up to $6 \mathrm{~mJ}$ in a regenerative amplifier. The four-pass amplification system cooled by liquid nitrogen boosted the energy up to $180 \mathrm{~mJ}$ (preamplifier) 


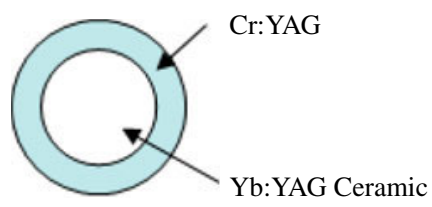

Figure 5. Edge-cladding Yb:YAG ceramic to improve the ASE suppression.

and $3.05 \mathrm{~J}$ (master amplifier) respectively. In future work, the optical transmission, pump and cooling structure will be further optimized to obtain a much higher energy output. In particular, an edge-cladding Yb:YAG ceramic (Figure 5) will be designed to improved the ASE suppression. Cr:YAG, which is commonly used for passive Q-switching, will be used as the edge-cladding material ${ }^{[22]}$.

\section{Acknowledgements}

The authors gratefully acknowledge the support of the Knowledge Innovation Project of the Chinese Academy of Sciences and the National Natural Science Foundation of China (No. 61008020).

\section{References}

1. J. C. Chanteloup and D. Albach, IEEE Photonics J. 3, 245 (2011).

2. T. Y. Fan, IEEE J. Quantum Electron. 29, 1457 (1993).

3. W. Koechner, Solid-state Laser Engineering (Springer-Verlag, Berlin, 1999).

4. M. Siebold, M. Hornung, R. Boedefeld, S. Podleska, S. Klingebiel, C. Wandt, F. Krausz, S. Karsch, R. Uecker, A. Jochmann, J. Hein, and M. C. Kaluza, Opt. Lett. 33, 2770 (2008).

5. F. Friebel, F. Druon, J. Boudeile, D. N. Papadopoulos, M. Hanna, P. Georges, P. Camy, J. L. Doualan, A. Benayad, R. Moncorgé, C. Cassagne, and G. Boudebs, Opt. Lett. 34, 1474 (2009).
6. G. Q. Xie, D. Y. Tang, L. M. Zhao, L. J. Qian, and K. Ueda, Opt. Lett. 32, 2741 (2007).

7. J. Kong, D. Y. Tang, C. C. Chan, J. Lu, K. Ueda, H. Yagi, and T. Yanagitani, Opt. Lett. 32, 247 (2007).

8. T. S. Rutherford, W. M. Tulloch, S. Sinha, and R. L. Byer, Opt. Lett. 26, 986 (2001).

9. Y. Akahane, M. Aoyama, K. Ogawa, K. Tsuji, S. Tokita, J. Kawanaka, H. Nishioka, and K. Yamakawa, Opt. Lett. 32, 1899 (2007).

10. D. S. Sumida and T. Y. Fan, Opt. Lett. 20, 2384 (1995).

11. D. J. Ripin, J. R. Ochoa, R. L. Aggarwal, and T. Y. Fan, Opt. Lett. 29, 2154 (2004).

12. K. H. Hong, J. T. Gopinath, D. Rand, A. M. Siddiqui, S. W. Huang, E. Li, B. J. Eggleton, J. D. Hybl, T. Y. Fan, and F. X. Kärtner, Opt. Lett. 35, 1752 (2010).

13. A. Bayramian, J. Armstrong, G. Beer, R. Campbell, B. Chai, R. Cross, A. Erlandson, Y. Fei, B. Freitas, R. Kent, J. Menapace, W. Molander, K. Schaffers, C. Siders, S. Sutton, J. Tassano, S. Telford, C. Ebbers, J. Caird, and C. Barty, J. Opt. Soc. Am. B 25, B57 (2008).

14. A. Lucianetti, T. Novo, B. Vincent, D. Albach, and J. C. Chanteloup, Proc. SPIE 8080 (2011).

15. T. Gonçalvès-Novo, D. Albach, B. Vincent, M. Arzakantsyan, and J.-C. Chanteloup, Opt. Express 21, 855 (2013).

16. R. Yasuhara, T. Kawashima, T. Sekine, T. Kurita, T. Ikegawa, O. Matsumoto, M. Miyamoto, H. Kan, H. Yoshida, J. Kawanaka, M. Nakatsuka, N. Miyanaga, Y. Izawa, and T. Kanabe, Opt. Lett. 33, 1711 (2008).

17. C. Wandt, S. Klingebiel, M. Siebold, Z. Major, J. Hein, F. Krausz, and S. Karsch, Opt. Lett. 33, 1111 (2007).

18. M. Hornung, R. Bödefeld, M. Siebold, A. Kessler, M. Schnepp, R. Wachs, A. Sävert, S. Podleska, S. Keppler, J. Hein, and M. C. Kaluza, Appl. Phys. B 101, 93 (2010).

19. R. L. Aggarwal, D. J. Ripin, J. R. Ochoa, and T. Y. Fan, J. Appl. Phys. 98, 103514 (2005).

20. T. Y. Fan, D. J. Ripin, R. L. Aggarwal, J. R. Ochoa, B. Chann, M. Tilleman, and J. Spitzberg, IEEE J. Sel. Top. Quantum Electron. 13, 448 (2007).

21. X. H. Lu, J. F. Wang, X. Li, Y. E. Jiang, W. Fan, and X. Ch. Li, Opt. Lett. 9, 111401 (2011).

22. J. Dong, K.-i. Ueda, A. Shirakawa, H Yagi, T. Yanagitani, and A. A. Kaminskii, Opt. Express 15, 14516 (2007). 